\title{
PSEUDO-ANOSOV MAPS AND CONTINUUM THEORY
}

\author{
ALFONSO ARTIGUE
}

(Communicated by Nimish Shah)

\begin{abstract}
In the hyperspace of subcontinua of a compact surface we consider a second order Hausdorff distance. This metric space is compactified in such a way that the stable foliation of a pseudo-Anosov map is naturally identified with a hypercontinuum. We show that negative iterates of a stable arc converge to this hypercontinuum in the considered metric. Some dynamical properties of pseudo-Anosov maps, as topological mixing and the density of stable leaves, are generalized for cw-expansive homeomorphisms of pseudo-Anosov type on compact metric spaces.
\end{abstract}

\section{INTRODUCTION}

In order to motivate some of the ideas in this paper let us start with an example. Let $f: T^{2} \rightarrow T^{2}$ be the Anosov diffeomorphism of the two dimensional torus defined by $f(x, y)=(2 x+y, x+y)$. The local stable sets are arcs and they form the stable foliation of $f$. Take $A^{s} \subset T^{2}$ a stable arc. In this case $f^{n}\left(A^{s}\right)$ converges to $T^{2}$ in the Hausdorff metric as $n \rightarrow-\infty$. The Hausdorff metric dist $_{H}$ between compact subsets is recalled in equation (11) below. Considering $f^{-1}$ we obtain a similar result for an unstable arc $A^{u}$. Then, $f^{-n}\left(A^{s}\right)$ and $f^{n}\left(A^{u}\right)$ have a common limit, the torus, as $n \rightarrow+\infty$ in the Hausdorff metric. This is what we find if we consider limits of stable and unstable arcs in the Hausdorff metric, but these limits do not show that the arcs are winding around the surface in different directions. The purpose of this paper is to apply well known constructions in continuum theory to find a space where the limits of $f^{-n}\left(A^{s}\right)$ and $f^{n}\left(A^{u}\right)$, as $n \rightarrow+\infty$, are the stable and the unstable foliations (two different points) interpreted in this space. If $X$ is a compact metric space, then we denote by $\mathcal{C}(X)$ the set of compact connected subsets of $X$. We will consider the following metric on $\mathcal{C}(X)$.

Definition 1.1 (Second order Hausdorff distance). For $A_{1}, A_{2} \in \mathcal{C}(X)$ the distance $\operatorname{dist}_{\tilde{H}}\left(A_{1}, A_{2}\right)$ is the minimal value of $r>0$ satisfying: for $\{i, j\}=\{1,2\}$ and every subcontinuum $C \subset A_{i}$ there is a subcontinuum $D \subset A_{j}$ such that $\operatorname{dist}_{H}(C, D) \leq r$.

In general $\left(\mathcal{C}(X), \operatorname{dist}_{\tilde{H}}\right)$ is not compact, as shown in Example 2.4. In this paper we consider a compactification $\tilde{\mathcal{C}}(X)$ of $\left(\mathcal{C}(X), \operatorname{dist}_{\tilde{H}}\right)$ via hypercontinua, that is, continua in $\left(\mathcal{C}(X), \operatorname{dist}_{H}\right)$. We will see that some hypercontinua are naturally associated to foliations. In this way we will be able to see how a continuum is stretching in the direction of the corresponding foliation. These ideas will be extended for cw-expansive homeomorphisms on a compact metric space. Recall from

Received by the editors August 2, 2015 and, in revised form, June 13, 2016 and August 25, 2016.

2010 Mathematics Subject Classification. Primary 37B45. 
3, 4. that a homeomorphism $f: X \rightarrow X$ is cw-expansive if there is $\delta>0$ such that if $A \subset X$ is connected with more than one point, then there is $n \in \mathbb{Z}$ such that $\operatorname{diam}\left(f^{n}(A)\right)>\delta$.

This paper is organized as follows. In Section 2 well known facts from continuum theory are recalled and examples are given. The reader should consult 2 for more on these topics. In Section 3 we apply the constructions obtained in the study of pseudo-Anosov maps from a viewpoint of continuum theory. In Theorems 3.5] and 3.10 we show that negative iterates of stable arcs converge to the hypercontinuum associated to the stable foliation. In Section 4 some properties of pseudo-Anosov maps are generalized to arbitrary cw-expansive homeomorphisms of a continuum.

\section{HyperContinuA}

Let $(X$, dist) be a continuum, i.e., a compact connected metric space. Denote by $\mathcal{C}(X)$ the set of continua contained in $X$, that is,

$$
\mathcal{C}(X)=\{A \subset X: A \text { is compact and connected }\} .
$$

In $\mathcal{C}(X)$ the Hausdorff metric is defined as

$$
\operatorname{dist}_{H}\left(A_{1}, A_{2}\right)=\inf \left\{\varepsilon>0: A_{1} \subset B_{\varepsilon}\left(A_{2}\right) \text { and } A_{2} \subset B_{\varepsilon}\left(A_{1}\right)\right\},
$$

where $B_{\varepsilon}(x)$ is the open ball of radius $\varepsilon$ centered at $x \in X$, and if $A \subset X$, then $B_{\varepsilon}(A)=\bigcup_{x \in A} B_{\varepsilon}(x)$. It is known that $\left(\mathcal{C}(X)\right.$, $\left.\operatorname{dist}_{H}\right)$ is compact and arc-connected [2, given that $X$ is compact and connected. The space $\mathcal{C}(X)$ is usually called the hyperspace of $X$.

Now we will consider a second level of abstraction. Denote $\mathcal{C}^{2}(X)=\mathcal{C}(\mathcal{C}(X))$. In $\mathcal{C}^{2}(X)$ we consider the Hausdorff distance $\operatorname{dist}_{H^{2}}$ induced by the metric $\operatorname{dist}_{H}$ of $\mathcal{C}(X)$. We have again that $\left(\mathcal{C}^{2}(X)\right.$, dist $\left._{H^{2}}\right)$ is compact and arc-connected. The elements of $\mathcal{C}^{2}(X)$ will be called hypercontinua.

Each element $A \in \mathcal{C}(X)$ can be seen in $\mathcal{C}^{2}(X)$ in two natural ways. First, as a singleton $\{A\} \in \mathcal{C}^{2}(X)$. Second, the way that will be explored in this paper, we can consider $\mathcal{C}(A) \in \mathcal{C}^{2}(X)$, i.e., the set formed by $A$ and its subcontinua. Let us plainly define a map representing this idea. Define $i: \mathcal{C}(X) \rightarrow \mathcal{C}^{2}(X)$ a $\sharp^{1}$

$$
i(A)=\left\{A^{\prime} \in \mathcal{C}(X): A^{\prime} \subset A\right\} .
$$

We say that $i(A) \in \mathcal{C}^{2}(X)$ is the hypercontinuum associated to the continuum $A \in \mathcal{C}(X)$.

Now we will state some properties of the map $i$. It is easy to prove that $i: \mathcal{C}(X) \rightarrow$ $\mathcal{C}^{2}(X)$ is injective. It is known that the map $i$ may not be continuous 2 Let us give an example showing this statement.

Example 2.1. Consider the square $X=[0,1] \times[0,1]$ with the Euclidean metric. Take $A$ and $A_{r}$ in $\mathcal{C}(X)$ as in Figure 1. We have that $\operatorname{dist}_{H}\left(A_{r}, A\right) \rightarrow 0$ as $r \rightarrow 0$. Let us show that the distance between $i(A)$ and $i\left(A_{r}\right)$ is bounded away from zero. In $\mathcal{C}^{2}(X)$ we consider the metric $\operatorname{dist}_{H^{2}}$ induced by $\operatorname{dist}_{H}$ in $\mathcal{C}(X)$. We have that $\operatorname{dist}_{H^{2}}\left(i(A), i\left(A_{r}\right)\right)<\varepsilon$ if and only if for each element of $i(A)$ there is an element of $i\left(A_{r}\right)$ that is $\varepsilon$-close, in the metric dist $_{H}$, to the element of $i(A)$, and conversely. This follows by the definition of the Hausdorff metric for the hypercontinua $i(A)$ and $i\left(A_{r}\right)$. Now notice that the set $A$ is formed by two arcs; call $A_{v}$ the vertical one.

\footnotetext{
${ }^{1}$ In fact, $i(A)=\mathcal{C}(A)$.

${ }^{2}$ In [2], a space $X$ whose induced map $i$ is continuous is called $C^{*}$-smooth. There, our map $i$ is called $C^{*}$.
} 


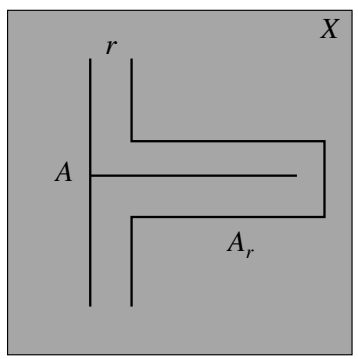

Figure 1. Subcontinua of the square $X$. The parameter $r$ denotes the width of the corridor determined by $A$ and $A_{r}$.

Then, $i(A)$ and $i\left(A_{r}\right)$ are far apart because there is no subcontinuum of $A_{r}$ being close to $A_{v}$, independently of how small $r$ is. This proves that $i$ is not continuous.

Via the injective map $i$ we define the second order Hausdorff metric on $\mathcal{C}(X)$ as

$$
\operatorname{dist}_{\tilde{H}}\left(A_{1}, A_{2}\right)=\operatorname{dist}_{H^{2}}\left(i\left(A_{1}\right), i\left(A_{2}\right)\right) .
$$

As the reader can check, this is exactly the metric given in Definition 1.1

Proposition 2.2. It holds that

$$
\operatorname{dist}_{H}\left(A_{1}, A_{2}\right) \leq \operatorname{dist}_{\tilde{H}}\left(A_{1}, A_{2}\right)
$$

for all $A_{1}, A_{2} \in \mathcal{C}(X)$.

Proof. It is direct from the definitions. However we give the details. Given $\varepsilon>$ $\operatorname{dist}_{\tilde{H}}\left(A_{1}, A_{2}\right)$ we will show that $\operatorname{dist}_{H}\left(A_{1}, A_{2}\right)<\varepsilon$. If $\operatorname{dist}_{\tilde{H}}\left(A_{1}, A_{2}\right)<\varepsilon$ and $x \in A_{1}$ (similar for $A_{2}$ ), then there is a continuum $A \subset A_{2}$ such that $\operatorname{dist}_{H}(\{x\}, A)<\varepsilon$. Then, for every $a \in A$ we have that $\operatorname{dist}(x, a)<\varepsilon$. Consequently, $\operatorname{dist}_{H}\left(A_{1}, A_{2}\right)<$ $\varepsilon$.

Define

$$
\tilde{\mathcal{C}}(X)=\mathcal{C}(X) \cup[\operatorname{clos}(i(\mathcal{C}(X))) \backslash i(\mathcal{C}(X))] .
$$

The closure is taken in $\left(\mathcal{C}^{2}(X)\right.$, $\left.\operatorname{dist}_{H^{2}}\right)$. The metric $\operatorname{dist}_{\tilde{H}}$ is extended to $\tilde{\mathcal{C}}(X) 3$ The space $\left(\tilde{\mathcal{C}}(X), \operatorname{dist}_{\tilde{H}}\right)$ is a compactification of $\left(\mathcal{C}(X), \operatorname{dist}_{\tilde{H}}\right)$ by adding some limit hypercontinua. Let us give some examples to illustrate these concepts (see [2] for more on this subject).

Example 2.3 (Hypercontinua of the interval). Consider the interval $X=[0,1]$ with its usual metric and define the triangle $T=\{(a, b) \in[0,1] \times[0,1]: a \leq b\}$. The continua in $X$ are intervals $[a, b] \subset[0,1]$. Therefore we can identify the hyperspace $\mathcal{C}([0,1])$ with $T$ using the map $[a, b] \in \mathcal{C}([0,1]) \mapsto(a, b) \in T 4$ In this case the map $i$ is continuous. Then, $\mathcal{C}([0,1])$ is compact with the metric $\operatorname{dist}_{\tilde{H}}$ and $\tilde{\mathcal{C}}([0,1])=$ $\mathcal{C}([0,1])$. In this case no hypercontinuum is needed in the compactification.

The situation is different for the circle.

\footnotetext{
${ }^{3}$ The reader can consider $\tilde{\mathcal{C}}(X)=\operatorname{clos}(i(\mathcal{C}(X))$. However, we wish to think of $\tilde{\mathcal{C}}(X)$ as a set extending $\mathcal{C}(X)$.

${ }^{4}$ In this case the hyperspace is two dimensional, a triangle. It is known that if $X$ has topological dimension greater than 1, then the topological dimension of the hyperspace is infinite [2].
} 
Example 2.4 (Hypercontinua of the circle). Let $X=S^{1}$ be the circle $\mathbb{R} / \mathbb{Z}$ with its usual metric and orientation. The subcontinua of $X$ can be parameterized by its starting point and its length. Length zero means singleton and length 1 is $X$ independent of the starting point. Therefore, $\mathcal{C}\left(S^{1}\right)$ can be identified with a cone (topologically a disc) obtained by collapsing a boundary of the cylinder $X \times[0,1]$. Let us now obtain a model for $\tilde{\mathcal{C}}\left(S^{1}\right)$. Let us first show that the map $i$ is not continuous. Consider a sequence of $\operatorname{arcs} A_{n} \subset X$ with a common starting point $s \in X$ and length $1-1 / n$. We have that the limit of $i\left(A_{n}\right)$ in $\tilde{\mathcal{C}}(X)$ is the marked hypercircle

$$
S_{s}^{1}=\left\{A \in \mathcal{C}\left(S^{1}\right): s \text { is not in the interior of } A\right\} .
$$

In the Hausdorff metric, the sequence $A_{n}$ converges to $S^{1}$, but $i\left(A_{n}\right)$ does not converge to $i\left(S^{1}\right)$. Then, $i$ is not continuous. In this case, the space $\left(\mathcal{C}\left(S^{1}\right), \operatorname{dist}_{\tilde{H}}\right)$ is compactified by adding the marked hypercircles. In this way we obtain that $\tilde{\mathcal{C}}\left(S^{1}\right)$ has two components. One component is a cylinder that is the union of the arcs of length less than 1 compactified with the marked hypercircles. The other component is the singleton $\left\{S^{1}\right\}$. In Figure 2 the spaces are illustrated.
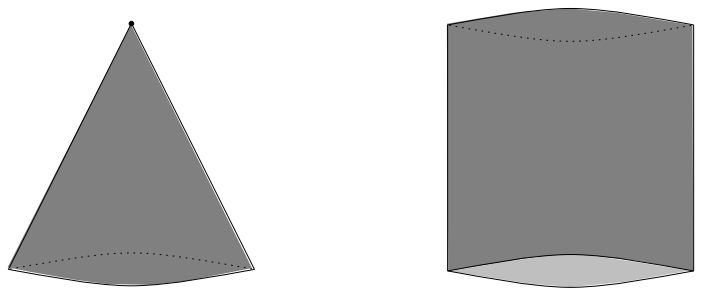

Figure 2. Left: the hyperspace $\mathcal{C}\left(S^{1}\right)$ of the circle. Right: the space $\tilde{\mathcal{C}}\left(S^{1}\right)$; notice the singleton component above the cylinder.

\section{Pseudo-Anosov maps}

Let $f: S \rightarrow S$ be a pseudo-Anosov map of the compact orientable surface $S$ with empty boundary. Denote by $F^{s}$ and $F^{u}$ the stable and the unstable singular foliations of $f$, respectively. We assume that $F^{s}$ and $F^{u}$ have a finite number of singularities, being each one an $n$-prong with $n \geq 1$. On the surface we consider a flat metric with conical points at the singularities of the foliations. In this case the curvature is concentrated at the singularities. See, for example, [5] for more on this topic. For an $n$-prong singularity $p$ the curvature at $p$ equals

$$
\kappa(p)=(2-n) \pi .
$$

For an arbitrary cone point the curvature at $p$ is $\kappa(p)=2 \pi-\theta(p)$ where $\theta(p)$ is the angle of the cone. In our case $\theta(p)$ is an integer multiple of $\pi$. The Gauss-Bonnet Theorem for flat surfaces with conical points [7, Theorem 17.1] gives us

$$
\sum_{p \in \operatorname{Sing}(S)} \kappa(p)=2 \pi \chi(S)
$$

where $\operatorname{Sing}(S)$ is the set of conical points of the surface and $\chi(S)$ denotes its Euler characteristic. 
3.1. Non-negative curvature. In this section we will suppose that the curvature is non-negative at each singularity, i.e., $\kappa(p) \geq 0$ for all $p \in \operatorname{Sing}(S)$. The general case is considered in the next section. The non-negative curvature condition implies that $\chi(S) \geq 0$ and consequently the surface is the sphere or the torus. In the case of the torus there are no singular points, and we will assume that $f$ is an Anosov diffeomorphism on a flat torus. In the case of the sphere there must be 4 singularities, $p_{1}, p_{2}, p_{3}, p_{4}$, with $\kappa\left(p_{i}\right)=\pi$. This follows by the Gauss-Bonnet Theorem because the Euler characteristic of the sphere equals 2. A singularity of curvature $\pi$ is usually called 1-prong.

Remark 3.1 (Double branched cover). Let $F$ be a singular foliation of the sphere $S$. At each regular point $x \in S^{*}=S \backslash \operatorname{Sing}(S)$ we have two orientations $o_{x}^{+}$and $o_{x}^{-}$ of the leaf of $x$. Define $\tilde{S}^{*}=\left\{\left(x, o_{x}^{\sigma}\right): x \in S^{*}, \sigma=+,-\right\}$. The canonical projection $\pi: \tilde{S}^{*} \rightarrow S^{*}$, given by $\pi\left(x, o_{x}^{\sigma}\right)=x$, induces a branched covering $\pi: \tilde{S} \rightarrow S$ where $\tilde{S}$ is a compact surface without boundary obtained from $\tilde{S}^{*}$ by adding finitely many points. Since $\tilde{S}$ has no singularity it must be a torus.

Now we will associate a hypercontinuum to the stable and unstable singular foliations of $f$. We start by defining

$$
\mathcal{C}\left(F^{\sigma}\right)=\left\{A \in \mathcal{C}(S): A \text { is contained in a leaf of } F^{\sigma}\right\}
$$

for $\sigma=s, u$. Notice that the elements of $\mathcal{C}\left(F^{\sigma}\right)$ are arcs or singletons (trivial arcs). This is because we are not considering $n$-prong singularities with $n>2$.

Proposition 3.2. The sets $\mathcal{C}\left(F^{\sigma}\right), \sigma=s, u$, are connected in $\left(\mathcal{C}(S)\right.$, $\left.\operatorname{dist}_{H}\right)$.

Proof. Take two stable $\operatorname{arcs} A_{1}, A_{2} \in \mathcal{C}\left(F^{\sigma}\right)$. Suppose that $\gamma_{i}:[0,1] \rightarrow S$ are continuous curves, $i=1,2$, such that $\gamma_{i}([0,1])=A_{i}$. Define $\alpha_{j}:[0,1] \rightarrow \mathcal{C}\left(F^{\sigma}\right)$, for $j=1,2,3$, such that $\alpha_{1}(t)=\gamma_{1}([0,1-t]), \alpha_{3}(t)=\gamma_{2}([0, t])$ and $\alpha_{2}(t)=\{\beta(t)\}$ where $\beta:[0,1] \rightarrow S$ is a continuous curve from $\gamma_{1}(0)$ to $\gamma_{2}(0)$. The concatenation of $\alpha_{1}, \alpha_{2}$ and $\alpha_{3}$ is a continuous curve from $A_{1}$ to $A_{2}$ inside $\mathcal{C}\left(F^{\sigma}\right)$. This proves that $\mathcal{C}\left(F^{\sigma}\right)$ is arc-connected, in particular connected.

Let us show that $\mathcal{C}\left(F^{\sigma}\right)$ is not closed in $\mathcal{C}(S)$. Given an arc $A \in \mathcal{C}\left(F^{\sigma}\right)$ denote by len $(A)$ its length with respect to the flat Riemannian metric of the surface. Take a sequence $A_{n} \in \mathcal{C}\left(F^{\sigma}\right)$ such that $\operatorname{len}\left(A_{n}\right)=n$. It is easy to see that $A_{n}$ has no convergent subsequence in $\mathcal{C}\left(F^{\sigma}\right)$. Therefore, it is not closed. Define

$$
\tilde{F}^{\sigma}=\operatorname{clos}\left(\mathcal{C}\left(F^{\sigma}\right)\right)
$$

where the closure is taken in $\left(\mathcal{C}(S), \operatorname{dist}_{H}\right)$. From Proposition 3.2 and the fact that the closure of a connected set is connected, we know that $\tilde{F}^{\sigma} \in \mathcal{C}^{2}(S)$; i.e., $\tilde{F}^{\sigma}$ is a hypercontinuum.

Proposition 3.3. It holds that $\tilde{F}^{\sigma}=\mathcal{C}\left(F^{\sigma}\right) \cup\{S\}$ for $\sigma=s, u$.

Proof. Let $A_{n}$ be a sequence in $\mathcal{C}\left(F^{\sigma}\right)$. If $A_{n}$ is convergent $\mathcal{C}(S)$ but its limit is not in $\mathcal{C}\left(F^{\sigma}\right)$, then $\operatorname{len}\left(A_{n}\right) \rightarrow \infty$. In this case it is easy to prove that $\lim _{n \rightarrow+\infty} \operatorname{dist}_{H}\left(A_{n}, S\right)=0$, which finishes the proof.

Since $\mathcal{C}\left(F^{\sigma}\right) \subset \mathcal{C}(S)$ we have that $\tilde{F}^{\sigma} \in \tilde{\mathcal{C}}(S)$. In the next theorem we will show that $f^{-n}\left(A^{s}\right)$ converges to $\tilde{F}^{s}$ in the metric dist $\tilde{H}$ if $A^{s}$ is a non-trivial stable arc. For its proof we need the following simple lemma. 
Lemma 3.4. If $x, y \in S$ and $A_{x}$ is a stable arc starting at $x$, then there is a stable arc $A_{y}$ starting at $y$ such that $\operatorname{dist}_{H}\left(A_{x}, A_{y}\right) \leq \operatorname{dist}(x, y)$ and $\operatorname{len}\left(A_{y}\right) \leq \operatorname{len}\left(A_{x}\right)$.

Proof. In the case of the torus we can consider its Lie group structure. Therefore, the stable $\operatorname{arc} A_{y}$ is obtained as $A_{x}+y-x$, a translation of $A_{x}$. We obtain $\operatorname{len}\left(A_{y}\right)=\operatorname{len}\left(A_{x}\right)$.

In the case of the sphere we can consider the double branched covering explained in Remark 3.1. Then, $A_{y}$ is the projection of the corresponding arc in the torus constructed in the previous paragraph. Notice that if a stable arc contains a singularity, then its projection may be a shorter arc.

Theorem 3.5. If $f: S \rightarrow S$ is a pseudo-Anosov map with non-negative curvature, then

$$
\lim _{n \rightarrow+\infty} \operatorname{dist}_{\tilde{H}}\left(f^{-n}\left(A^{s}\right), \tilde{F}^{s}\right)=0
$$

for every non-trivial stable arc $A^{s} \in C\left(F^{s}\right)$. Similarly for $A^{u} \in C\left(F^{u}\right)$.

Proof. Let $A^{s}$ be a non-trivial stable arc. Recall that

$$
\operatorname{dist}_{\tilde{H}}\left(f^{n}\left(A^{s}\right), \tilde{F}^{s}\right)=\operatorname{dist}_{H^{2}}\left(i\left(f^{n}\left(A^{s}\right)\right), \tilde{F}^{s}\right)
$$

and notice that $i\left(f^{n}\left(A^{s}\right)\right) \subset \tilde{F}^{s}$ for all $n \in \mathbb{Z}$. We will show that for all $\varepsilon>0$ there is $n_{0}$ such that if $n>n_{0}$ and $A_{1}$ is a stable arc, then there is a stable arc $A_{2} \subset f^{-n}\left(A^{s}\right)$ such that $\operatorname{dist}_{H}\left(A_{1}, A_{2}\right)<\varepsilon$. Given $\varepsilon>0$, take $l>0$ such that if $A$ is a stable arc and $\operatorname{len}(A) \geq l$, then $\operatorname{dist}_{H}(A, S)<\varepsilon / 2$. Given a stable arc $A^{s}$ consider $n_{0}$ such that

$$
\operatorname{len}\left(f^{-n}\left(A^{s}\right)\right)>3 l
$$

for all $n \geq n_{0}$. Consider an arbitrary stable arc $A_{1}$. If $\operatorname{len}\left(A_{1}\right) \geq l$, then

$$
\operatorname{dist}_{H}\left(A_{1}, f^{-n}\left(A^{s}\right)\right) \leq \operatorname{dist}_{H}\left(A_{1}, S\right)+\operatorname{dist}_{H}\left(S, f^{-n}\left(A^{s}\right)\right) \leq \varepsilon / 2+\varepsilon / 2=\varepsilon .
$$

In this case, the stable arc $A_{2}$ mentioned above is $A_{2}=f^{-n}\left(A^{s}\right)$. Now assume that $\operatorname{len}\left(A_{1}\right)<l$. Suppose that $A_{1}$ starts at $x$. Since $\operatorname{len}\left(f^{-n}\left(A^{s}\right)\right)>3 l$ we can take three disjoint subarcs, each one having length $l$. Denote by $A_{*}^{s}$ the one in the middle. Take $y \in A_{*}^{s}$ such that $\operatorname{dist}(x, y)<\varepsilon$. From Lemma 3.4 we know that there is a stable arc $A_{y}$ such that $\operatorname{len}\left(A_{y}\right) \leq l$ and $\operatorname{dist}_{H}\left(A_{y}, A_{1}\right)<\varepsilon$. Since $y \in A_{*}^{s}$ and $\operatorname{len}\left(A_{y}\right) \leq l$ we have that $A_{y} \subset f^{-n}\left(A^{s}\right)$. Therefore, taking $A_{2}=A_{y}$ the proof ends.

Remark 3.6. Let us explain why Theorem 3.5 is not true for a pseudo-Anosov map with negative curvature. For $\delta>0$ define the local stable set of $p$ as

$$
W_{\delta}^{s}(p)=\left\{q \in S: \operatorname{dist}\left(f^{n}(p), f^{n}(q)\right) \leq \delta \text { for all } n \geq 0\right\} .
$$

If $f$ has an $n$-prong singular point $p \in S$, with $n>2$, then $W_{\delta}^{s}(p)$ of $p$ is the union of $n$ arcs starting at $p$. We can see that no non-trivial stable arc $A^{s}$ can be arbitrarily close to $W_{\delta}^{s}(p)$. Since $W_{\delta}^{s}(p) \in \tilde{F}^{s}$, we have that $\operatorname{dist}_{\tilde{H}}\left(f^{-n}\left(A^{s}\right), \tilde{F}^{s}\right)$ is bounded away from zero. Also, note that Lemma 3.4 is not true if there are $n$-prongs with $n \geq 3$. In the next section we explain how to adapt Theorem 3.5 for pseudo-Anosov maps with singularities of negative curvature. 
3.2. Arbitrary curvature. Let $f: S \rightarrow S$ be a pseudo-Anosov map of a compact surface $S$. As before, denote by $F^{s}$ and $F^{u}$ the stable and the unstable singular foliations of $f$, respectively. In this section we consider $n$-prong singularities with any $n=1,3,4, \ldots$ In relation to Remark 3.6 we introduce the following concept.

Definition 3.7. A rectangle $R \subset S$ is a product box if there is a homeomorphism $\varphi:[0,1] \times[0,1] \rightarrow R$ sending vertical arcs to stable arcs and horizontal arcs to unstable arcs. We say that an arc of the foliation $F^{\sigma}$ is accessible if it is contained in a product box.

Remark 3.8. For a generalized pseudo-Anosov with non-negative curvature, as in the previous section, every stable continuum is an accessible arc. The local stable set of a 3-prong singularity is a non-accessible stable arc, because it is not even an arc. For an $n$-prong singularity $p$ with $n>3$ there are stable arcs that are not accessible: take the union of two stable arcs starting at $p$ but in non-consecutive sectors.

Define

$$
\mathcal{C}_{a}\left(F^{\sigma}\right)=\left\{A \in \mathcal{C}(S): A \text { is an accessible arc of } F^{\sigma}\right\}
$$

for $\sigma=s, u$.

Proposition 3.9. The sets $\mathcal{C}_{a}\left(F^{\sigma}\right), \sigma=s, u$, are connected in $\left(\mathcal{C}(S)\right.$, dist $\left.{ }_{H}\right)$.

Proof. Similar to the proof of Proposition 3.2. Note that singletons are accessible stable (and unstable) arcs.

Define the hypercontinuum $\tilde{F}_{a}^{\sigma}=\operatorname{clos}\left(\mathcal{C}_{a}\left(F^{\sigma}\right)\right)$. As in the previous section, it holds that $\tilde{F}_{a}^{\sigma}=\mathcal{C}_{a}\left(F^{\sigma}\right) \cup\{S\}$. The arguments for the following result are similar to those in the proof of Theorem 3.5. However, Lemma 3.4 is not true in the present case and some care is needed.

Theorem 3.10. If $f$ is a pseudo-Anosov map, then

$$
\lim _{n \rightarrow+\infty} \operatorname{dist}_{\tilde{H}}\left(f^{-n}\left(A^{s}\right), \tilde{F}_{a}^{s}\right)=0
$$

for every non-trivial accessible stable arc $A^{s} \in C_{a}\left(F^{s}\right)$.

Proof. Fix $\varepsilon>0$ given. Take $l_{0}>0$ such that if $A^{s}$ is a stable $\operatorname{arc}$ with $\operatorname{len}\left(A^{s}\right) \geq l_{0}$, then $\operatorname{dist}_{H}\left(A^{s}, S\right)<\varepsilon / 2$. Consider a family of closed product boxes $R_{1}, \ldots, R_{k}$ of the foliations such that for every stable arc $A^{s}$ with $\operatorname{len}\left(A^{s}\right) \leq l_{0}$ there is a box $R_{j}$ containing $A^{s}$. These boxes can be obtained from negative iterates of an arbitrary cover of product boxes. Also assume that for every stable arc $A^{s}$ contained in some box $R_{j}$ and every point $x \in R_{j}$ there is a stable arc $A^{\prime}$ such that $x \in A^{\prime} \subset R_{j}$ and $\operatorname{dist}_{H}\left(A^{\prime}, A^{s}\right)<\varepsilon$. Take $l_{1}>0$ such that every stable arc of length $l_{1}$ intersects every box $R_{j}$. Also assume that no stable arc contained in a box $R_{j}$ is longer than $l_{1}$.

Take $A_{1}, A_{2}$ stable arcs and assume that $\operatorname{len}\left(A_{2}\right)>\max \left\{3 l_{1}, l_{0}\right\}$. We will find a stable arc $A_{3} \subset A_{2}$ such that $\operatorname{dist}_{H}\left(A_{1}, A_{3}\right)<\varepsilon$. If $\operatorname{len}\left(A_{1}\right) \geq l_{0}$, then

$$
\operatorname{dist}_{H}\left(A_{1}, A_{2}\right) \leq \operatorname{dist}_{H}\left(A_{1}, S\right)+\operatorname{dist}_{H}\left(S, A_{2}\right)<\varepsilon / 2+\varepsilon / 2=\varepsilon .
$$

If len $\left(A_{1}\right)<l_{0}$, then $A_{1}$ is contained in a box, say $R_{j^{*}}$. Divide $A_{2}$ into three arcs of equal length and take the one in the middle, $A_{2}^{*}$ with $\operatorname{len}\left(A_{2}^{*}\right)>l_{1}$. We know that $A_{2}^{*}$ intersects $R_{j^{*}}$. Take $x \in A_{2}^{*} \cap R_{j^{*}}$. The maximal stable arc of $x$ in the box $R_{j^{*}}$ 
contains a stable arc $A_{3}$ such that $\operatorname{dist}_{H}\left(A_{3}, A_{1}\right)<\varepsilon$. By construction, $A_{3} \subset A_{2}$ and the proof ends.

An analogous result holds for an unstable arc $A^{u} \in C_{a}\left(F^{u}\right)$.

It is known that pseudo-Anosov maps are topologically mixing and that every stable leaf is dense in the surface; see for example 1 for the proofs. Also, pseudoAnosov maps are cw-expansive. This essentially follows by the behavior of product boxes. In the next section we generalize these properties for an arbitrary cwexpansive homeomorphism on a continuum.

\section{Hypercontinua AND CW-EXPANSIVITY}

Let $f: X \rightarrow X$ be a cw-expansive homeomorphism of the compact metric space (X, dist). We say that $A \in \mathcal{C}(X)$ is a stable continuum if $\operatorname{diam}\left(f^{n}(A)\right) \rightarrow 0$ as $n \rightarrow+\infty$. An unstable continuum is a stable continuum for $f^{-1}$. Following [4] we define

and $W_{f}^{u}=W_{f-1}^{s}$.

$$
W_{f}^{s}=\left\{A \in \mathcal{C}(X): \lim _{n \rightarrow+\infty} \operatorname{diam}\left(f^{n}(A)\right)=0\right\}
$$

Remark 4.1. The sets $W_{f}^{\sigma}, \sigma=s, u$, will play the role of $\mathcal{C}\left(F^{\sigma}\right)$ in Section 3.1

For an arbitrary homeomorphism $g: Y \rightarrow Y$ of a metric space and $y \in Y$ we define, as usual, the $\omega$-limit set $\omega_{g}(y)$ as the set of the points $x \in Y$ for which there is a sequence of integers $n_{k} \rightarrow+\infty$ such that $g^{n_{k}}(y) \rightarrow x$. The $\alpha$-limit set is defined as $\alpha_{g}(y)=\omega_{g^{-1}}(y)$.

Define $\tilde{f}: \tilde{\mathcal{C}}(X) \rightarrow \tilde{\mathcal{C}}(X)$ as the natural homeomorphism induced by $f$. The following result represents the idea that, for a cw-expansive homeomorphism (as a pseudo-Anosov map), the negative iterates of a stable continuum and the positive iterates of an unstable continuum wind in $X$ in different directions.

Theorem 4.2. If $f$ is cw-expansive, then $\alpha_{\tilde{f}}\left(A^{s}\right)$ and $\omega_{\tilde{f}}\left(A^{u}\right)$ are disjoint for every non-trivial $A^{s} \in W_{f}^{s}$ and $A^{u} \in W_{f}^{u}$.

Proof. By contradiction suppose that there is $A \in \alpha_{\tilde{f}}\left(A^{s}\right) \cap \omega_{\tilde{f}}\left(A^{u}\right)$. By definition we know that there is $n_{k} \rightarrow+\infty$ such that $\tilde{f}^{n_{k}}\left(A^{u}\right) \rightarrow A$ as $k \rightarrow+\infty$ in the metric $\operatorname{dist}_{\tilde{H}}$. Also, there is $m_{k} \rightarrow-\infty$ such that $\tilde{f}^{m_{k}}\left(A^{s}\right) \rightarrow A$ as $k \rightarrow+\infty$. We know that $A \in \tilde{\mathcal{C}}(X)$.

First suppose that $A \in \mathcal{C}(X)$. Given any $\varepsilon>0$ we can take a subcontinuum $A^{\prime}$ of $A$ with $\operatorname{diam}\left(A^{\prime}\right)<\varepsilon$. By definition of $\operatorname{dist}_{\tilde{H}}$, for each $k \geq 1$ there is a subcontinuum $B_{k}^{\prime}$ of $\tilde{f}^{n_{k}}\left(A^{u}\right)$ making a convergent sequence $\operatorname{dist}_{H}\left(B_{k}^{\prime}, A^{\prime}\right) \rightarrow 0$. If $\varepsilon$ is small, then we obtain that $A^{\prime}$ is an unstable continuum (this can be proved applying [3, Corollary 2.4]). In the same way we have that $A^{\prime}$ is stable. Since $f$ is cw-expansive we conclude that $A^{\prime}$ is a singleton. This implies that $A$ is a singleton.

Assume now that $A \in \tilde{\mathcal{C}}(X) \backslash \mathcal{C}(X)$. Arguing as in the previous paragraph, we can prove that there is $\varepsilon>0$ such that if $A^{\prime} \in A$ and $\operatorname{diam}\left(A^{\prime}\right)<\varepsilon$, then $A^{\prime}$ is a singleton. Since $A$ is a $\operatorname{dist}_{\tilde{H}}$-limit of continua we have that $A$ is a singleton. This contradicts that $A \in \omega_{\tilde{f}}\left(A^{u}\right)$ with $A^{u}$ non-trivial, and the proof ends.

Example 4.3. Consider $f: X \rightarrow X$ as the solenoid attractor that is explained in for example [6]. It is the inverse limit of the circle map $z \mapsto z^{2}$. As noticed by Kato [3. Proposition 3.1], the solenoid attractor is positive cw-expansive 
(every non-trivial continuum is expanded in positive iterates). In this case we have that stable continua are singletons. It is known (see [2]) that the map $i$ is continuous in this case 5 As a consequence, for every unstable non-trivial arc $A^{u}$ it holds that

$$
\lim _{n \rightarrow+\infty} \operatorname{dist}_{\tilde{H}}\left(f^{n}\left(A^{u}\right), X\right)=0 .
$$

Proposition 4.4. If $X$ is a continuum, then $W_{f}^{s}$ and $W_{f}^{u}$ are connected with respect to dist $_{H}$.

Proof. Given a stable continuum $A^{s}$ it is known that there is a continuous curve $\gamma:[0,1] \rightarrow \mathcal{C}(X)$ such that $\gamma(0)=A^{s}, \gamma(1)$ is a singleton while $\gamma(t) \subset A^{s}$ for all $t \in[0,1]$; see [2]. Now, since the space $X$ is connected we have that $\{\{x\}: x \in X\}$ is connected with respect to $\operatorname{dist}_{H}$. Since every singleton $\{x\}$ is a stable continuum we conclude that $W_{f}^{s}$ is connected with respect to $\operatorname{dist}_{H}$.

Define

$$
\tilde{F}^{\sigma}=\operatorname{clos}\left(W_{f}^{\sigma}\right)
$$

for $\sigma=s, u$. The closure is considered in $\mathcal{C}(X)$ with $\operatorname{dist}_{H}$. We say that a homeomorphism $f$ is topologically mixing if for any non-empty open sets $U$ and $V$ of $X$, there is a natural number $N>0$ such that $U \cap f^{n}(V) \neq \emptyset$ for all $n \geq N$.

The following result is similar to [4, Theorem 3.5] for fully expansive homeomorphisms.

Theorem 4.5. If $f$ is a cw-expansive homeomorphism of the continuum $X$ and

$$
\left.\begin{array}{l}
\lim _{n \rightarrow-\infty} \operatorname{dist}_{\tilde{H}}\left(f^{n}\left(A^{s}\right), \tilde{F}^{s}\right)=0, \\
\lim _{n \rightarrow+\infty} \operatorname{dist}_{\tilde{H}}\left(f^{n}\left(A^{u}\right), \tilde{F}^{u}\right)=0
\end{array}\right\} \forall \text { non-trivial } A^{\sigma} \in W_{f}^{\sigma}, \sigma=s, u,
$$

then $f$ is topologically mixing.

Proof. If $X$ is a singleton the result is trivial; therefore, we assume that $X$ has positive topological dimension. By [3, Proposition 2.5] we know that, changing $f$ with $f^{-1}$ if needed, there is a non-trivial continuum $A^{s} \in W_{f}^{s}$. Then, $\lim _{n \rightarrow+\infty} \operatorname{dist}_{\tilde{H}}\left(f^{-n}\left(A^{s}\right), \tilde{F}^{s}\right)=0$. By definition, for all $\varepsilon>0$ there is $n_{0}$ such that if $n>n_{0}$, then $\operatorname{dist}_{\tilde{H}}\left(f^{-n}\left(A^{s}\right), \tilde{F}^{s}\right)<\varepsilon$. In particular, for every singleton $\{x\}$ in $\tilde{F}^{s}$ there is $y \in f^{-n}\left(A^{s}\right) \cap B_{\varepsilon}(x)$. Therefore, $f^{-n}\left(A^{s}\right)$ converges to $X$ in $\operatorname{dist}_{H}$. This is true for every non-trivial $A^{s} \in W_{f}^{s}$. Analogously, for a non-trivial unstable continuum $A^{u} \in W_{f}^{u}$ we have that $f^{n}\left(A^{u}\right)$ converges to $X$ in $\operatorname{dist}_{H}$.

Given two open subsets $U$ and $V$ of $X$, take $n$ such that $f^{-n}\left(A^{s}\right)$ intersects $U$. Now take a non-trivial subcontinuum $A_{*}^{s} \subset f^{-n}\left(A^{s}\right) \cap U$. Since $\operatorname{dist}_{H}\left(f^{-j}\left(A_{*}^{s}\right), X\right) \rightarrow$ 0 , there is $j_{0}$ such that for all $j>j_{0}$ it holds that $f^{-j}\left(A_{*}^{s}\right) \cap V \neq \emptyset$. This proves that $f$ is topologically mixing.

Remark 4.6. For a cw-expansive homeomorphism of a continuum $X$ satisfying (2) we have:

(1) for every non-trivial stable continuum $A^{s}$, $\operatorname{dist}_{H}\left(f^{-n}\left(A^{s}\right), X\right) \rightarrow 0$ as $n \rightarrow$ $+\infty$ (analogous for a non-trivial unstable continuum). This was proved in the first paragraph of the proof of Theorem 4.5.

\footnotetext{
${ }^{5}$ In terms of 2 , the solenoid is $C^{*}$-smooth.
} 
(2) if there is a non-trivial stable continuum, then there is $\delta>0$ such that for each $x \in X$ there is a stable continuum $A^{s}$ such that $\operatorname{diam}\left(A^{s}\right)=\delta$ and $x \in A^{s}$. This follows from the previous property and the techniques of [3. Section 2].

For $x \in X$ define

$$
C W_{f}^{\sigma}(x)=\left\{y \in X: \exists A \in W_{f}^{\sigma} \text { such that } x, y \in A\right\}
$$

for $\sigma=s, u$.

Proposition 4.7. If $X$ is a continuum and $f$ is a cw-expansive homeomorphism satisfying (2), then $C W_{f}^{\sigma}(x)$ is dense in $X$ for all $x \in X$ and $\sigma=s, u$.

Proof. From Remark 4.6, we can assume that there is $\delta>0$ such that for every $y \in X$ there is a stable continuum $A$ such that $\operatorname{diam}(A)=\delta$ and $y \in A$. Pick $x \in X$. For each $n \geq 0$ consider $A_{n}$ a stable continuum with $\operatorname{diam}\left(A_{n}\right)=\delta$ and $f^{n}(x) \in A_{n}$. Define $A_{n}^{\prime}=f^{-n}\left(A_{n}\right)$. For each $n \geq 0$ we have that $A_{n}^{\prime} \subset C W_{f}^{s}(x)$. Then, $\bigcup_{n \geq 0} A_{n}^{\prime}$ is dense in $X$. Since $\bigcup_{n \geq 0} A_{n}^{\prime} \subset C W_{f}^{\sigma}(x)$ the proof ends.

As we have seen, several dynamical properties of pseudo-Anosov maps can be deduced from the cw-expansivity and condition (2). It would be interesting to characterize pseudo-Anosov maps among cw-expansive homeomorphisms of compact surfaces. For example: is every topologically mixing cw-expansive surface homeomorphism conjugate to a pseudo-Anosov map?

\section{ACKNOWLEDGEMENT}

The author thanks the referee for the kind comments and suggestions that helped improve the exposition.

\section{REFERENCES}

[1] Benson Farb and Dan Margalit, A primer on mapping class groups, Princeton Mathematical Series, vol. 49, Princeton University Press, Princeton, NJ, 2012. MR2850125

[2] Alejandro Illanes and Sam B. Nadler Jr., Hyperspaces, fundamentals and recent advances, Monographs and Textbooks in Pure and Applied Mathematics, vol. 216, Marcel Dekker, Inc., New York, 1999. MR1670250

[3] Hisao Kato, Continuum-wise expansive homeomorphisms, Canad. J. Math. 45 (1993), no. 3, 576-598, DOI 10.4153/CJM-1993-030-4. MR.1222517

[4] Hisao Kato, Concerning continuum-wise fully expansive homeomorphisms of continua, Topology Appl. 53 (1993), no. 3, 239-258, DOI 10.1016/0166-8641(93)90119-X. MR.1254870

[5] Howard Masur and John Smillie, Quadratic differentials with prescribed singularities and pseudo-Anosov diffeomorphisms, Comment. Math. Helv. 68 (1993), no. 2, 289-307, DOI 10.1007/BF02565820. MR 1214233

[6] Clark Robinson, Dynamical systems: Stability, symbolic dynamics, and chaos, 2nd ed., Studies in Advanced Mathematics, CRC Press, Boca Raton, FL, 1999. MR.1792240

[7] Richard Evan Schwartz, Mostly surfaces, Student Mathematical Library, vol. 60, American Mathematical Society, Providence, RI, 2011. MR2809109

Departamento de Matemática y Estadistica del Litoral, Universidad de la República, Gral. Rivera 1350, 50000 Salto, Uruguay

E-mail address: artigue@unorte.edu.uy 